\section{BIOMATERIALS}

\section{Primed by polymers}

Biochemistry 51, 2276-2288 (2012)

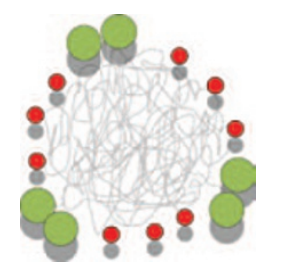

Polyhydroxybutyrate (PHB) synthase (PhaC) from Ralstonia eutropha synthesizes $\mathrm{PHB}$ polymers to store carbon. Studies with recombinant $\mathrm{PhaC}$ have shown that the polymerization reaction proceeds with a slow initiation phase and a fast elongation phase. Though initiation can be increased by the addition of the coenzyme A ester of a hydroxybutyrate trimer, the slow kinetics suggested that another factor primes $\mathrm{PhaC}$ for activity in vivo. To search for this factor, Cho et al. created a Strep2-tagged PhaC that could be directly purified from the host organism. Isolation of PhaC after $4 \mathrm{~h}$ or $24 \mathrm{~h}$ of growth yielded a highly active species, suggesting the authors had succeeded in isolating the primed enzyme. SDS-PAGE analysis identified the known $\mathrm{PHB}$-associated protein $\mathrm{PhaP} 1$ as purifying together with $\mathrm{PhaC}$, but this protein slowed initiation in in vitro assays. Size-exclusion chromatography to monitor enzyme oligomerization unexpectedly identified a soluble, high-molecular-weight species that contained $\mathrm{PhaP} 1, \mathrm{PhaC}$ and $\mathrm{PHB}$ in addition to the expected monomer and dimer of PhaC. As the soluble complex was highly active and was not formed with mutant $\mathrm{PhaC}$, the authors concluded that PHB itself is the likely priming factor. These results led to a 'modified micelle' model, in which a $\mathrm{PhaC}$ dimer is covalently attached to a single $\mathrm{PHB}$ chain and several PhaP1s associate with this species in soluble form prior to conversion to insoluble granules.

$C G$

\section{STRUCTURAL BIOLOGY}

\section{Versatile conformation}

Nat. Struct. Mol. Biol., published online 11 March 2012, doi:10.1038/nsmb.2253

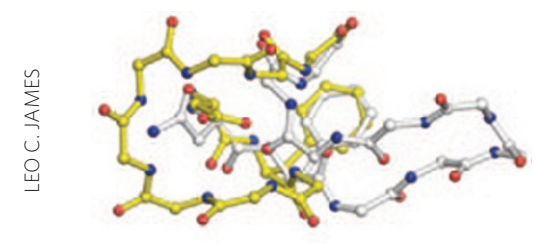

The Rhesus macaque TRIMCyp (RhTC) antiviral protein binds and restricts multiple lentiviruses, whereas the homologous protein cyclophilin A (CypA) targets only HIV-1. Previous work showed that two mutations in RhTC gave rise to a new binding-site conformation. Caines et al. discover that RhTC adopts multiple new conformations and this structural versatility allows RhTC to target different viruses. The authors crystallized the RhTC CypA domain bound to either HIV-1 or HIV-2 capsid and observed that the two

APOPTOSIS

\title{
Sphingolipid cofactors
}

Apoptosis depends upon BCL-2 proteins for mitochondrial outer membrane permeabilization (MOMP) and cytochrome $c$ release. The mitochondrial outer membrane also interacts with other organelles at loci known to regulate lipid biosynthesis. Although sphingolipid metabolism is known to regulate apoptosis, the relationship between sphingolipids and BCL-2 proteins is not well understood. Chipuk et al. develop a biochemical protocol for isolating mitochondria from heavy membrane fractions (HMFs) and apply it to show that higher doses of an activator of BAK, a proapoptotic BCL-2 protein, are required to induce cytochrome $c$ release from mitochondria than from HMFs. Adding back microsomal components removed during purification restored sensitivity. Peptide analysis of the microsomes revealed the presence of two neutral sphingomyelinases (N-SMases). Exogenous $\mathrm{N}$-SMases cooperated with proapoptotic factors and mitochondria to promote MOMP. The authors confirmed that N-SMases were present in heterotypic mitochondriaassociated membranes. Reconstitution of sphingolipid metabolism or inclusion of sphingolipid metabolites revealed that BAK cooperates with sphingosine-1-phosphate and that BAX cooperates with hexadecenal to promote cytochrome $c$ release. The authors validated and extended these findings in a TNF-induced model of apoptosis in mammalian cells. Taken together, these data suggest that heterotypic mitochondrial membrane interactions are necessary to provide the appropriate sphingolipid environment for MOMP to proceed efficiently. Future studies will be needed to understand mechanistically how sphingolipids affect the activation of proapoptotic BCL-2 proteins.

RhTC structures are substantially different. NMR analyses revealed that the loop in which the two RhTC mutations reside is no longer constrained by hydrogen bonds; it is highly mobile compared to CypA. The authors validated the role of the loop in the recognition of multiple viruses by reversing its structural flexibility. They introduced a cysteine to force the formation of a disulfide bridge, and as a result they locked the position of the loop and the conformation of the active site. Isothermal titration calorimetry and X-ray crystallography showed that the constrained RhTC lost the ability to recognize HIV-2. Reduction of the disulfide bond reversed the constraint and allowed RhTC to bind multiple viruses. This study reveals how an antiviral protein acquired an adaptable binding site and the ability to target multiple viruses. $A C$

EPIGENETICS

\section{Docking PCNA}

Mol. Cell, published online 1 March 2012, doi:10.1016/j.molcel.2012.01.019

Post-translational modifications, such as methylation or acetylation of lysine residues in histones, are essential regulators of chromatin structure and gene expression. For example, acetylation of Lys56 of histone $\mathrm{H} 3$ (H3K56ac) is abundant in yeast, in which it is associated with nucleosomal assembly processes. Yu et al. now show that monomethylation of this same lysine residue (H3K56me1) in human cells serves as an important mark for recruiting the DNA replication apparatus. Western blotting and MS analysis identified monomethylated, but not dimethylated or trimethylated, forms of $\mathrm{H} 3 \mathrm{~K} 56$ in HeLa cells. The histone lysine methyltransferase G9a installed H3K56me1 in vitro and was required for the maintenance of this mark in cells. H3K56me1 modifications were not associated with transcriptionally active chromatin or heterochromatin; instead, the disruption of H3K56 monomethylation led to impaired DNA replication. Chromatin immunoprecipitation experiments revealed that $\mathrm{H} 3 \mathrm{~K} 56 \mathrm{me} 1$ was required for recruitment of proliferating cell nuclear antigen (PCNA) - the 'DNA clamp' protein that encircles DNA during replication-to chromatin during the G1 phase of the cell cycle. This binding interaction was confirmed by showing that H3K56me1-modified synthetic peptides bind PCNA in vitro and selectively pull it down from nuclear extracts. These results define a histone mark that facilitates assembly of the DNA replication machinery and highlight how subtle structural modifications of chromatin can be linked to diverse regulatory roles. 